\title{
Zusammenhang zwischen Sprache und Mathematik
}

Gesamtüberblick: Sprache ist für mathematische Lehr- und Lernprozesse relevant, sowohl in Bezug auf sprachlich-kommunikative als auch auf inhaltliche Aspekte. Die Bedeutung, die Sprache für den Mathematikunterricht in den letzten Jahren dazugewonnen hat, ist anhand von internationalen Vergleichs- und Folgestudien zu erklären (Abschnitt 2.1). Anhand dieser Arbeiten sind die sprachlichen Voraussetzungen von Lernenden unter der Perspektive von heterogenen Lerngruppen ein substanzieller Gesichtspunkt, der im Mathematikunterricht mitgedacht werden muss (Abschnitt 2.2). Sprache kann funktional beschrieben werden, was bedeutet, dass die Sprache im Kontext von fachlichen Lehr- und Lernprozessen differenziert wird, je nachdem, wofür sie verwendet wird (Abschnitt 2.3). Die funktionale Nutzung von Sprache lässt sich auf einer kognitiven Ebene als Werkzeug des Denkens - und einer kommunikativen Ebene - als Werkzeug der Vermittlung - betrachten (Abschnitt 2.3.1). Werden diesbezüglich empirische Befunde zur kognitiven Funktion analysiert, deren Bedeutung für den Mathematikunterricht nicht zwangsläufig so klar ist wie die der kommunikativen Funktion (Abschnitt 2.3.2), wird deutlich, dass eine Analyse der sprachlichen Funktion für den Mathematikunterricht bedeutend ist. In der vorliegenden Arbeit wird auf theoretische Aspekte der funktionalen Grammatik zurückgegriffen. Dahingehend ist die Betrachtung der funktionalen Elemente von Sprache zu diskutieren, die Parallelen in der Unterscheidung zwischen kognitiver und kommunikativer Funktion zeigen (Abschnitt 2.3.3). Neben den Funktionen, die die Bedeutung der Sprache im Mathematikunterricht unterstreichen, werden drei weitere Aspekte von Sprache betrachtet (Abschnitt 2.4). So ist Sprache Gegenstand des Lehrens und Lernens von Mathematik, beispielsweise durch die Fachbegriffe, die gelernt werden (Abschnitt 2.4.1). Daneben nimmt Sprache als Lernmedium eine Rolle für den Mathematikunterricht ein, da sie zur Vermittlung der Inhalte dient

D. Bednorz, Sprachliche Variationen von mathematischen Textaufgaben, Bielefelder Schriften zur Didaktik der Mathematik 5, https://doi.org/10.1007/978-3-658-33003-3_2 
(Abschnitt 2.4.2). Durch die beiden zuvor geschilderten Aspekte ergeben sich Implikationen für den dritten Aspekt. Die sprachlichen Voraussetzungen haben Einfluss auf den Lernerfolg im Mathematikunterricht. Geringe sprachliche Voraussetzungen der Lernenden können sich damit als Lernhindernis darstellen (Abschnitt 2.4.3).

\subsection{Bedeutung von Sprache für den Mathematikunterricht}

Sprache hat als Untersuchungsgegenstand für das Lernen mathematischer Inhalte in der Mathematikdidaktik eine lange Tradition. Einerseits existieren interpretative Ansätze, die insbesondere mit dem Namen Heinrich Bauersfeld in Verbindung gebracht werden (H. Maier, 1991). Diese Ansätze fokussieren die Analyse und Deutung von Sprache im Kontext von Aushandlungsprozessen. Andererseits wird die Fachsprache als abstrakte Formalsprache betrachtet und insbesondere der Umgang von Lernenden mit den Symbolen der Mathematik beschrieben (Freudenthal, 1973, 1983).

Die Aktualität des Themas Sprache hat in den letzten Jahren an Relevanz dazu gewonnen. Dies ist anhand von internationalen empirischen Studien wie PISA (Programme for International Student Assessment) sowie TIMSS (Trends in International Mathematics and Science Study) und dem stärkeren Fokus auf die Leistungsheterogenität der Lernenden nachzuvollziehen. Die verstärkte Betrachtung der Heterogenität von Lernenden ist differenziert zu erklären. Erstens haben sich die sozialen Bedingungen, beispielsweise Migration und Urbanisierung, verändert und zweitens hat die UN-Behindertenrechtskonvention maßgeblich inklusive Ansätze zur Beschulung für verschiedene Schulformen beeinflusst und damit die vielfältigen Eingangsvoraussetzungen der Lernenden weiter differenziert (Overwien \& Prengel, 2007; Pijl et al., 1997; UNESCO, 2009). Die mathematikspezifischen, leistungsbezogenen Heterogenitätsaspekte sind mindestens genauso zentral wie die sozialen und bildungspolitischen Einflüsse auf die Leistungsdisparitäten im Bildungsverlauf. Dies implizieren die angesprochenen internationalen Vergleichsstudien wie die PISA-Studie, die seit dem Jahr 2000 erhoben wird. Die PISA-Studie ist im Hinblick auf die aktualisierte Betrachtung von Sprache auch im Fachunterricht entscheidend, da hierin die unterschiedlichen Leistungen von monolingualen im Vergleich zu nicht monolingualen Lernenden belegt wurden (Klieme et al., 2010). Darauf aufbauend, belegen weitere Studien den Einfluss von sprachlichen Konstrukten auf die mathematische Leistung (vgl. Abschnitt 2.4.3). Sowohl in der Forschung als auch in der Praxis wurde 
damit Sprache als Element des Lehrens und Lernens von Mathematik verstärkt betrachtet.

\subsection{Sprachliche Heterogenität}

Wie im vorherigen Kapitel geschildert, ist die Heterogenität ein bedeutender Grund dafür, dass Sprache im Mathematikunterricht an Relevanz gewinnt. Dies liegt an den unterschiedlichen (heterogenen) sprachlichen Voraussetzungen von Lernenden.

Heterogenität ist jedoch in der Diskussion um diese Voraussetzungen nicht neu. Bereits in früheren pädagogischen Auseinandersetzungen bei Comenius, Humboldt, Herbart und weiteren zum Teil reformpädagogischen Ansätzen existierte eine thematische Auseinandersetzung mit den ungleichen Ausgangsbedingungen (Ehlers, 2004). Diese Ansätze verweisen auf die Verschiedenheit von Lernenden im Denken und Handeln (Tillmann, 2004).

Unter linguistischer Betrachtung lässt sich der erste Teil des Wortes Heterogenität aus dem griechischen Wort heteros (andersartig) ableiten und impliziert damit die Fragestellung: Anders als was? Beim Begriff der Heterogenität handelt es sich um ein soziales Phänomen. Das Wort ist ein relationaler Terminus, der das Endergebnis eines normativen Vergleichs von Bezügen darstellt (Frauendorfer, 2011; Hagedorn, 2010; Wittek, 2013). Dieser Vergleich beschränkt sich nicht auf die kognitiven Dimensionen der Lernenden, hat jedoch immer eine Bezugsnorm, mit der verglichen wird.

Für den Kosmos Schule beschreibt Tillmann (2004) eine lange Tradition der Fixierung auf die Homogenität von Lerngruppen. Mittlerweile ist die Betrachtung der Heterogenität jedoch manifester Bestandteil der sozialen und schulischen Realität geworden und kennzeichnet, dass es in der Praxis zur Distanzierung von der Fixierung auf die Homogenität der Lerngruppen kommt (Wittek, 2013). Durch die breite Akzeptanz ist der erfolgreiche Umgang mit Heterogenität zu einem Merkmal für Unterrichtsqualität geworden (König et al., 2015).

Die Voraussetzungen, in denen sich Lernende in schulisch relevanten Kontexten unterscheiden, sind mannigfaltig (Wittek, 2013). Beispielsweise differenzieren sich Lernende in Merkmalen wie Schulleistung, Herkunft, Geschlechterrollen, sozialen Milieus, körperlichen Bedingungen und Sprache (Bos et al., 2004).

In Verbindung mit der Heterogenität steht der Begriff der Inklusion. Der Zusammenhang zwischen diesen Termini ergibt sich, da Inklusion als eine positive Auseinandersetzung mit der Heterogenität von Lernenden definiert werden kann, die dabei alle Dimensionen der Heterogenität dieser Personen betont (Hinz, 
2009). Positiv bedeutet hierbei, dass Heterogenität insbesondere als Chance und nicht als Hindernis für das Lernen verstanden wird. Damit entwickelt sich Inklusion von einem bildungspolitischen Terminus zu einer didaktischen Kategorie, anhand derer sich das Gelingen eines - im Fall dieser Arbeit - sprachintegrierten Mathematikunterrichts unter der Perspektive von Inklusion als Qualitätsmerkmal beschreiben lässt.

Für Forschung und Praxis ist es relevant $\mathrm{zu}$ fokussieren, in welchen Aspekten sich Lernende unterscheiden und welche Einflüsse diese Unterschiede auf das Lern- und Leistungsverhalten im Mathematikunterricht haben. Nur so können passende adaptive didaktische Maßnahmen entwickelt und erforscht werden, die es den Lernenden mit unterschiedlichen Voraussetzungen ermöglichen, sowohl auf schulischer als auch auf gesellschaftlicher Ebene zu partizipieren.

Wendt et al. (2016) zeigen in der internationalen Vergleichsstudie für Grundschulkinder TIMSS 2015, dass sich Leistungsdiskrepanzen durch Heterogenitätsmerkmale aufgrund von herkunftsbezogenen Unterschieden feststellen lassen. Für Lernende mit Migrationshintergrund zeigen sich signifikante Leistungsdisparitäten, die deutlicher ausfallen, wenn beide Elternteile im Ausland geboren sind (Wendt et al., 2016). Im Vergleich zum Erhebungszeitraum 2007 fallen diese jedoch im Jahr 2016 geringer aus. Damit scheint es erfolgreicher zu gelingen, Lernende mit heterogener Herkunft zu fördern (Wendt et al., 2016). Prenzel et al. (2013) stellen fest, dass sich Lernende mit bzw. ohne Zuwanderungshintergrund einerseits in PISA 2003, andererseits in PISA 2012 signifikant in der mathematischen Kompetenz unterscheiden. Es zeigt sich bei PISA 2012, dass sich die Unterschiede zwischen den Lernenden ohne Zuwanderungshintergrund und denen, die in der zweiten Generation in Deutschland leben, im Vergleich zu PISA 2003 reduziert haben (Prenzel et al., 2013). Die Tendenzen von PISA 2015 und TIMSS 2015 indizieren damit, dass die Bemühungen, die infolge der Studien erfolgten, Wirkung zeigen.

Des Weiteren belegen Prenzel et al. (2013), dass zusätzliche Indikatoren eine Rolle in der Mediation des Einflusses der Zuwanderungsgeschichte auf die mathematische Leistung einnehmen. Die Ergebnisse zeigen, dass der Zuwanderungshintergrund nicht alleinige Grundlage der Disparitäten in der Mathematikleistung ist und dass weitere Merkmale, beispielsweise der sozioökonomische Status oder die sprachlichen Kompetenzen, bei der Erklärung der Unterschiede relevant sind (Prenzel et al., 2013).

Im Kontext von sprachlichen Fähigkeiten als Merkmal der Lernenden belegen Prediger et al. (2015), dass die sprachliche Kompetenz im Vergleich zu anderen Merkmal von Lernenden in ihrer Studie die größte Bedeutung bei der Erklärung des Zusammenhangs mit der Mathematikleistung hat. In der Studie von Ufer et al. 
(2013) wird darauf hingewiesen, dass die sprachlichen Fähigkeiten besonders bei Aufgaben bedeutsam sind, deren Fokus auf konzeptuell-inhaltlichen Facetten (u. a. Sachaufgaben) liegt. So stellen Ufer et al. (2013) für den Lernzuwachs einen signifikanten Einfluss des Sprachstandes auf konzeptuell-inhaltliche Facetten auf inhaltlicher Ebene fest, der höher ist als der der kognitiven Grundfertigkeiten.

Das Desiderat aus den genannten Studien begründet den Fokus der Arbeit, der auf Sprache als einem zentralen Heterogenitätsmerkmal für das Lernen mathematischer Inhalte liegt. Folglich sind Unterrichtsqualität und der Lehr- sowie Lernerfolg im Mathematikunterricht substanziell von der Betrachtung von Sprache als Heterogenitätsmerkmal geprägt. Unter dieser Perspektive beschreiben Vollmer und Thürmann (2010, S. 21-22) das integrierte Konzept des Sprachlernens als Aufgabe der ganzen Schule unter der Prämisse „,der Entwicklung und Unterstützung eines akademischen Sprachgebrauchs im Fachunterricht“, mit dem Ziel, dass sprachliche Unterschiede durch Förderung auf einem hohen Niveau angeglichen werden können, um so Bildungserfolg zu ermöglichen.

\subsection{Funktionen von Sprache}

Um der sprachlichen Heterogenität im Mathematikunterricht gerecht zu werden, benötigt es einen theoretischen Rahmen, der dazu genutzt werden kann, Sprache im Fachunterricht zu fördern. Für den Mathematikunterricht bietet es sich an, unterschiedliche Funktionen von Sprache differenziert zu betrachten.

Überblick (Abschnitt 2.3): Die Betrachtung sprachlicher Funktionen auf kognitiver und kommunikativer Ebene zeigt zwei Perspektiven auf das Phänomen der Sprache in fachlichen Lehr- und Lernprozessen (Abschnitt 2.3.1). Der Einfluss der kommunikativen Funktion ist aufgrund des Vermittlungsaspektes unstrittig; unklar ist jedoch, ob und in welcher Weise die kognitive Funktion einen Effekt auf das Denken aufweist und ob dieser empirisch zu beobachten ist. Diese Fragestellung wird durch linguistische Studien adressiert, in denen der Einfluss auf das Denken und Sprechen analysiert wird (Abschnitt 2.3.2). Aufgrund des Forschungsfokus der vorliegenden Arbeit wird ergänzend zu der in Abschnitt 2.3.1 vorgestellten Unterscheidung in die kommunikative und kognitive Funktion die funktionale Differenzierung von Sprache nach der funktionalen Grammatik dargestellt (Abschnitt 2.3.3). 


\subsubsection{Kommunikative und kognitive Funktion}

Die Unterscheidung in eine kommunikative und eine kognitive Funktion von Sprache geht insbesondere auf H. Maier und Schweiger (1999) zurück. Sie verweisen mit der Betrachtung einer doppelten Funktion von Sprache auf Klix (1995), der diese Unterscheidung definiert.

Klix (1995) beschreibt die kommunikative Funktion aus einer biologischen Perspektive als eine Funktion, die die Vorreiterrolle der Sprache einnimmt und die insbesondere phonologische Aspekte der Sprache abdeckt. Dabei differenziert er die kommunikative Ebene in zwei Funktionen: zum einen die lautliche kommunikative Funktion und zum anderen die lernabhängige Kommunikation, die für ihn bereits ein Zwischenstadium von kognitiven Aspekten in der kommunikativen Funktion darstellt.

Menschen nutzen Sprache jedoch nicht nur zur lautlichen Kommunikation, sondern auch auf einer kognitiven Ebene als ein Beschreibungs- und Deutungsinstrument, das mit zusätzlichen semiotischen Systemen verknüpft werden kann, um das Denken zu erweitern, zu vereinfachen oder zu verkürzen (Tomasello, 2008). Für die kognitive Funktion von Sprache unterscheidet Klix (1995) vier unterschiedliche funktionale Ebenen:

1. die Funktion der Verdichtung des Informationstransports in der Kommunikation durch begriffliche Repräsentationen

2. die Fixierung neuer Wissensbereiche und deren begriffliche Durchdringung

3. das Teilen von Ergebnissen des individuellen Denkens als soziale Funktion des Denkens

4. die Wechselbeziehung zwischen Anschauungsbildern und begrifflichen Strukturen als kreatives Denken

Hiermit zeigt sich, dass eine Unterscheidung in kommunikative und kognitive Funktion nicht trivial ist, da beide Ebenen eng miteinander in Beziehung stehen, wie besonders im ersten (Informationstransport) und dritten (das Teilen) Punkt verdeutlicht wird. Mithin wird anhand dieser Beschreibung verdeutlicht, dass es problematisch ist, die kommunikative Funktion (separiert) zu betrachten, wenn beispielsweise sprachliche Hürden von Lernenden beschrieben werden. In dieser Hinsicht ist auch die von Meyer und Tiedemann (2017) hervorgehobene Erläuterung der verstärkenden Rolle der kommunikativen auf die kognitive Funktion, die H. Maier und Schweiger (1999) einbrachten, zu verstehen, und zwar als die Vermittlungsinstanz der kommunikativen Funktion für das Denken und damit auf die 
kognitive Funktion durch Aspekte der phonologischen bzw. lautlichen Kommunikation. So ist das Phänomen bekannt, dass Personen durch die Erklärung eines Sachverhaltes ebendiesen für sich genauer verstehen.

Gleichzeitig scheinen die unterschiedlichen kognitiven Funktionen (implizit) natürlicher Bestandteil des Lehrens und Lernens in der Mathematik und der mathematikdidaktischen Forschung zu sein - und dies auch ohne einen direkten Verweis auf die kognitive Funktion von Sprache. So betrachtet beispielsweise Freudenthal (1973) in seinen didaktischen Ausführungen den ersten Punkt der kognitiven Funktion der Verdichtung des Informationstransportes durch begriffliche Repräsentationen, indem er die Problematik der symbolischen Notation bei Lernenden diskutiert. Die sprachlichen Funktionen sind also bereits früh in die didaktische Perspektive genommen worden.

\subsubsection{Empirische Befunde zur kognitiven Funktion von Sprache}

Ausgehend von der geschilderten engen Verflechtung zwischen der Sprache auf der einen und dem fachlichen Inhalt auf der anderen Seite mit den Begriffen, Vorstellungen, Darstellungen und Kenntnissen von mathematischen (abstrakten) Objekten und den Operationen, die der Mathematikunterricht beinhaltet, sollen nachfolgend empirische Erkenntnisse zu der kognitiven Funktion von Sprache dargestellt werden.

Die Beziehung zwischen Sprache und der Kognition wurde in einer Reihe von linguistischen Studien durch den Vergleich von Sprachen untersucht. Aus mathematikdidaktischer Perspektive relevant sind solche Arbeiten, in denen die Verbindung zwischen Sprache und räumlichen sowie zeitlichen Strukturen fokussiert wird.

Levinson und Haviland (2009) beschreiben insbesondere die Unterschiede von Präpositionen (in, auf, zu, bei, über etc.) und Verben (drehen, fallen, erreichen etc.), die zur Bezeichnung von Bewegungsvorgängen und zu Ortsbeschreibungen verwendet werden, zwischen Englisch, Französisch und Deutsch im Vergleich zur Sprache der Maya (Tzeltal sowie Tzotzil). Dabei fehlt bei den Sprachen Tzeltal und Tzotzil eine deiktische Distinktion, d. h. Vorderseite, Rückseite, rechts, links. Entsprechende Wortgruppen sind jedoch zentral zur Beschreibung von Mustern, Strukturen und mathematischen Objekten wie geometrischen Figuren und Körpern, aber auch für angewandte Mathematik zur Beschreibung von realen Vorgängen (Levinson \& Haviland, 2009). 
Auch für andere Sprachen zeigt sich, dass sich die sprachlichen Systeme zur Beschreibung von räumlichen Situationen deutlich unterscheiden können und daneben die Wahrnehmung von Zeit beeinflussen (Boroditsky, 2000; Bowerman \& Choi, 2001; Casasanto et al., 2010). Gaby (2012) belegt, dass aus der Variabilität von Beschreibungssystemen auch unterschiedliche kognitive Handlungsmuster abgeleitet werden können. In der Untersuchung sollten Aborigines mit der Sprache Kuuk Thaayorre eine zeitliche Bildfolge auslegen. Die Aborigines legten die Bildfolge nach der Himmelsrichtung, wobei die Himmelsrichtung als frequentiertes, direktionales sprachliches Mittel in der Kuuk Thaayorre verwendet wurden. Die Sprache hat dementsprechend einen Effekt auf das Legen der Bildfolge. Die Studie von Boroditsky et al. (2008) deutet ebenfalls darauf hin, dass die Abfolge von zeitlichen Bildfolgen von der genutzten Sprache abhängt. So strukturierten Englisch sprechende und Deutsch sprechende Personen eine zeitliche Abfolge von links nach rechts, Arabisch sprechende legten die Karten tendenziell von rechts nach links, was der Schreibrichtung der jeweiligen Sprache entspricht.

Die empirischen Studien verweisen darauf, dass Kognition und Sprache eine enge Verbindung haben und sich je nach gesprochener Sprache in unterschiedlicher Weise ausprägen. Dahingehend ist neben der kommunikativen die kognitive Funktion von Sprache für Lehr- und Lernprozesse im Mathematikunterricht bedeutsam (Prediger, 2016). Bewusst wird die Relevanz der kognitiven Funktion bei einer Betrachtung der genannten Studien u. a. dann, wenn bedacht wird, dass zum Verständnis und zur Interpretation von Darstellungsformen im Mathematikunterricht Aspekte von Raum und Zeit essenziell sind, die durch Sprache vermittelt werden - und das sowohl auf kommunikativer als auch auf kognitiver Ebene.

\subsubsection{Funktionen im Sinne der funktionalen Grammatik}

Neben der für die mathematikdidaktische Forschungsliteratur üblichen Unterscheidung in die kommunikative und die kognitive Funktion von Sprache existieren weitere funktionale Unterscheidungsmöglichkeiten. Aufgrund des Fokus dieser Arbeit scheint die funktionale Beschreibung der funktionalen Grammatik bedeutsam.

Halliday (2014a) ist einer der bekanntesten Vertreter der funktionalen Grammatik und verwendet zur Analyse eine funktionale Beschreibung von Sprache. Er ordnet die Funktionen von Sprache in sein Konzept der Sprachvariationen ein, u. a. mit dem Begriff des Registers (vgl. Abschnitt 4.2) (Halliday, 2003a, 2004a, 
2004b, 2005, 2014a; H. Maier \& Schweiger, 1999). Unter Betrachtung der Analyse von Sprache nach einer soziolinguistischen und pragmatischen Orientierung wird die funktionale Beschreibung wie folgt begründet:

(W)hat are the basic functions of language, in relation to our ecological and social environment $[\ldots][\mathrm{w}] \mathrm{e}$ suggested two: making sense of our experience, and acting out our social relationships (Halliday, 2014a, S. 30).

Im Fokus der Halliday'schen Betrachtung von Funktionen von Sprache stehen dementsprechend sowohl die Bedeutungskonstruktion aus der Wahrnehmung als auch die soziale Beziehung und der damit einhergehende Austausch von Wahrnehmungen aus der Umwelt. Dieser theoretische Ansatz fokussiert eine konstruktive (sense making) und interaktionale (ecological) Basis und bietet damit einen naheliegenden Zugang zur funktionalen Untersuchung von Sprache bei Lehrund Lernprozessen im Fachunterricht. Halliday (2014a) definiert drei Typen von Basisfunktionen, die er im Kontext seiner Analyse als Metafunktionen bezeichnet. Er unterscheidet die:

1. ideelle (ideational) Metafunktion, die in erfahrungsgemäße (experiential) und logische (logical) Metafunktionen differenziert wird;

2. die interpersonale (interpersonal) Metafunktion und die

3. textuelle (textual) Metafunktion.

Erläuterungen zu den Metafunktionen: Die ideelle Metafunktion von Sprache verweist auf die Bedeutung von Sprache als Ressource zur Strukturierung und Konstruktion von menschlicher Erfahrung (Halliday, 2014a). In der Auseinandersetzung mit dieser Metafunktion verwendet Halliday bei seiner Erklärung der begrifflichen Fixierung von Sprache ähnliche Analogien wie Klix (1995, S. 34):

Begriffe sind Klassifizierungen von Objekten, Ereignissen oder Operationen nach den ihnen gemeinsamen (invarianten) Merkmalen. Sie werden im Gedächtnis gespeichert und bilden die Basis des klassifizierenden Erkennens: Ein Stamm, Zweige und Blätter oder Nadeln bilden den Begriff des Baumes. Und so für alle wahrnehmbaren Merkmalssätze.

In einer ähnlichen Weise beschreibt Halliday (2014a, S. 30) die ideelle (erfahrungsgemäße) Metafunktion: 
It is clear that language does - as we put it - construe human experience. It names things, thus construing them into categories; and then, typically, goes further and construes the categories into taxonomies, often using more names for doing so. So we have houses and cottages and garages and sheds, which are all kinds of building; strolling and stepping and marching and pacing, which are all kinds of walking; in, on, under, around as relative locations, and so on - and the fact that these differ from one language to another is a reminder that categories are in fact construed in language.

Halliday (2014a) sowie Klix (1995) beschreiben die Möglichkeit, unterschiedliche Erfahrungen zu gruppieren und Unterscheidungen durch die Wahl von sprachlichen Mitteln zu treffen. Dieses Alltagsphänomen, das bei unkomplizierten Begriffen wie Baum, Haus oder Auto beginnt, wird in der Mathematik und im Mathematikunterricht fortgeführt. So existieren Dreiecke, Vierecke, Fünfecke usw. Diese können in konvexe, konkave oder überschlagene Vielecke gruppiert werden. Dabei zeigt sich eine Vielfalt von Unterscheidungsmöglichkeiten, die durch die Sprache vermittelt werden, sowohl in der Realität als auch in der Mathematik.

Die begrifflichen Phänomene zur Strukturierung der Erfahrungswelt werden, als erfahrungsgemäße Metafunktionen charakterisiert, die durch die Verbindung miteinander jeder Form von Erscheinung Bedeutung verleihen können (Halliday, 2003b, 2014a). Halliday (2014a) führt den Vergleich von sprachlichen Funktionen weiter aus, indem er zusätzlich eine logische Metafunktion einführt. Die logische Metafunktion beschreibt die Möglichkeit, Begriffe miteinander durch komplexe grammatikalische Formen zu verbinden und in Beziehung zu setzen. Hierdurch lässt sich beispielsweise die Form der Blätter als Dreiecke klassifizieren, da eine sprachliche Verbindung zwischen Formen von Blättern und geometrischen Formen konstruiert werden kann. Die logische Verknüpfung von Begriffen ermöglicht eine erfahrungsunabhängige Verbindung von Wörtern, die zu einer formalisierten Sprache führt und $u$. a. die mathematische Fachsprache kennzeichnet.

Beide Ebenen der ideellen Metafunktion haben für den Mathematikunterricht Relevanz. Werden enaktive Objekte und Darstellungen betrachtet, verweisen diese auf die begriffliche Verwendung der erfahrungsgemäßen Metafunktionen. Es handelt sich um reale oder illustrierte Objekte, die beispielsweise zur Bildung von Vorstellungen zu (mathematischen) Objekten dienen können. Das bedeutet, dass durch die erfahrungsgemäßen Metafunktionen Begriffe entwickelt werden, die dann durch eine logische Verknüpfung weitergenutzt werden. Dahingehend stellt die operative Verknüpfung zwischen Sequenzen durch die logische Metafunktion, beispielsweise durch Konjunktionen wie wenn, als, während, Präpositionen wie $z u$, in, auf und Adverbien wie dann, eher, öfter die Erweiterung der begrifflichen, erfahrungsbezogenen Verwendung dar. Diese Metafunktion ermöglicht es, 
inhaltsbezogene bzw. begriffliche Erfahrungen durch Sprache auszutauschen, zu strukturieren und (neu) zu verknüpfen (Halliday, 2002).

Die interpersonale Metafunktion beschreibt den Gebrauch von Sprache als aktiven Prozess im persönlichen und gesellschaftlichen Austausch (Halliday, 2014a; Webster, 2019). Satzstrukturen bilden dabei nicht nur Begriffe oder Prozesse $\mathrm{ab}$ - wie in der ideellen Metafunktion - sondern sind auch Repräsentanten für die Form des Diskurses, was die Ausdrucksmöglichkeiten in abgegrenzten sozialen Gruppen und die persönliche Ausdrucks- und Entwicklungsfähigkeit erklärt (Halliday, 2002). Dabei verändern unterschiedliche Sprecherdiskurse den Rahmen des Austausches. So kann bei Fragen antizipiert werden, dass die intendierte gesprächsteilnehmende Person auf die gestellte Frage antwortet (Webster, 2019). Daneben können Satzstrukturen unterschiedliche Formen von sozialer Interaktion implizieren: So können Aussagen getroffen, Situationen bewertet oder es kann ein Appell gerichtet werden. Die Möglichkeiten, dabei zu interagieren, sind vielfältig (Halliday, 2014a; Webster, 2019).

Auch die interpersonale Metafunktion ist für den Mathematikunterricht bedeutsam. So ist insbesondere die Einführung von Operatoren als zentrale textuelle Sprachstrukturen wesentlich. Mit der Verwendung von Verben wurden insbesondere Fragen ersetzt, wodurch sich der Bezug zur interpersonalen Metafunktion ebenfalls verändert hat. Fragen wie Wie viele Teiler hat 120? wurden ersetzt durch Nenne die Anzahl der Teiler von 120. In den typischen Sprechakten bei Aufgabenstellungen werden insbesondere Verben im Imperativ verwendet, mit denen die lernende Person zur Aufgabenlösung bewegt werden soll (Lenz, 2015; Rehbein \& Kameyama, 2008). Durch die Umstellung auf Verboperatoren hat sich dementsprechend die Art der interpersonalen Metafunktion im Gebrauch von mathematischen Aufgabenstellungen grundlegend verändert. Daneben zeigt sich durch die Beispiele die Verbindung zwischen der funktionalen Betrachtung von Sprache und dem Mathematikunterricht.

Die dritte und letzte ist die textuelle Metafunktion. Ihr zentraler Gegenstand ist der Aufbau von sprachlichen Einheiten unter der Prämisse der Kohäsion und Kontinuität (Taboada, 2019). Der Zweck dieser Funktion ist die Möglichkeit der referenziellen Bezüge in einem Sprechakt. Kohäsion und Kontinuität sind für sprachliche Produkte so essenziell, dass es problematisch ist, sich beispielsweise nicht kohärente Texte vorzustellen, da das menschliche Gehirn sogar aus nicht plausiblen Texten versucht, einen Sinn zu konstituieren:

Coherence is such a fundamental property of texts and or our communication that it is difficult to conceive of completely incoherent texts. Consider the two invented examples in (1) and (2). [...] 
(1) I went home very late last night. At night, owls out and hunt. Harry Potter uses an owl to have his mail delivered. The mail was very erratic over the Christmas holidays. The holidays were too short, and short indeed is the paragraph.

(2) There were dark clouds in the sky today. However, it rained. (Taboada, 2019, S. 311)

Taboada (2019) konstruiert mit dem ersten Beispiel einen Textabschnitt, in dem kohäsive lexikalische Strukturen verwendet werden, beispielsweise: last night - at night, owls out-uses an owl oder this mail delivered-the mail was very. Die vereinzelnden Strukturen zeigen aber keinen kohärenten Bezug zueinander: So existiert kein Bezug der ersten night zu der zweiten night. Das zweite Beispiel demonstriert wiederum einen kurzen Textabschnitt, der nicht kohäsiv, aber kohärent ist. So ist die Implikation zwischen dark clouds und rained kohärent, jedoch existiert kein angemessener referenzieller Bezug, insbesondere aufgrund der Konjunktion however.

Damit stellt sich dar, dass die textuelle Metafunktion den Sprechakt in zweifacher Form in sinnvolle Texturen organisiert (Halliday, 2002; Webster, 2019). Zum einen werden sprachliche Botschaften um vorhergehend gemachte Informationen organisiert, zum anderen wird die Organisation davon bestimmt, was die sprechende Person durch die sprachliche Botschaft intendiert (Webster, 2019).

Auch bei der textuellen Metafunktion lassen sich konkrete Aspekte auf den Mathematikunterricht beziehen. Dabei kann im Fall von Textaufgaben beispielsweise davon ausgegangen werden, dass die Autorinnen oder Autoren der Aufgaben kohäsive Aufgaben stellen, indem zwischen den Sätzen Bezüge hergestellt werden (vgl. Abschnitt 5.2). Jedoch zeigen sich insbesondere für eingekleidete Textaufgaben, die nach Radatz und Schipper (2007) keinen Realitätsbezug aufweisen und alleine der Formulierung einer mathematischen Aufgabenkonstruktion dienen, keine kohärenten textlichen Strukturen - beispielsweise, wenn realitätsferne Textaufgaben gestellt werden und die Berechnung der Aufgabe sinnlos ist.

Resümee (Abschnitt 2.3): Die kommunikative und die kognitive Funktion sind einerseits für die mathematikdidaktische Forschung und andererseits für die Schulpraxis im Mathematikunterricht relevant. Die funktionale Beschreibung ermöglicht einen theoretischen Zugang zur Erklärung der Relevanz von Sprache auch für den Fach- bzw. Mathematikunterricht. Die kommunikative Funktion ist bedeutsam für Vermittlungsprozesse und die kognitive Funktion für Prozesse, die mit dem Denken zusammenhängen (Abschnitt 2.3.1). Die enge Verknüpfung zwischen Sprechen und Denken wird durch empirische Studien belegt (Abschnitt 2.3.2). Neben der Unterscheidung in eine kommunikative und eine 
kognitive Funktion ergeben sich weitere theoretische Möglichkeiten der Differenzierung. Unter anderem ist eine funktionale Unterscheidung nach der funktionalen Grammatik möglich. Hierbei wird in ideelle, interpersonale und textuelle Metafunktionen differenziert (Abschnitt 2.3.3).

Für den Mathematikunterricht werden neben der funktionalen Unterscheidung und Beschreibung von Sprache weitere Aspekte betrachtet. Diese werden in dem nachfolgenden Abschnitt 2.4 diskutiert.

\subsection{Aspekte}

Sprache hat nicht nur, wie im Abschnitt 2.3 dargestellt, auf einer funktionalen Basis für den Mathematikunterricht Relevanz. Es zeigt sich, dass Sprache auch für drei weitere sprachbezogene Aspekte (als Lerngegenstand, als Lernmedium und als Lernvoraussetzung bzw. Lernhindernis) bedeutsam ist. Während die funktionale Perspektive auf die theoretische Begründung abzielt, wie Sprache im Mathematikunterricht Einfluss auf Lehr- und Lernprozesse hat, fassen die Aspekte die Sprache als Lerngegenstand, Lernmedium und als Lernvoraussetzung bzw. Lernhindernis im Mathematikunterricht beschreiben, die generellen Kategorien zusammen, die hinsichtlich eines sprachintegrierten Mathematikunterrichts betrachtet werden sollten. Die Differenzierung dient der Sensibilisierung der Relevanz von Sprache in mathematikdidaktischen Prozessen und markiert damit, dass zur Steuerung und Ausgestaltung von Unterrichtsstunden nicht nur fachliche Lernziele, sondern stets auch die mit ihnen assoziierten Sprachhandlungen und Sprachmittel in die didaktischen Prozesse einbezogen werden müssen.

Überblick (Abschnitt 2.4): Der Mathematikunterricht vermittelt mathematische Inhalte. Diese sind grundlegend von (mathematischen) Begriffen und den dazugehörigen Sprachmitteln geprägt. Das bedeutet, dass Sprache selbst Gegenstand des mathematischen Lernens ist (Abschnitt 2.4.1). Sprache dient daneben als Vermittlungsinstanz. Sie wird dafür verwendet, mathematische Inhalte sprachlich zu transportieren. Dahingehend dient Sprache als Medium (Abschnitt 2.4.2). Aufgrund ihrer bereits erwähnten Bedeutung ist Sprache auch die Voraussetzung dafür, mathematische Inhalte zu lernen. Wenn diese sprachlichen Voraussetzungen nicht vorhanden sind, kann Sprache zum Lernhindernis für Lernende im Mathematikunterricht werden (Abschnitt 2.4.3). 


\subsubsection{Lerngegenstand}

Dass Sprache als Lerngegenstand verstanden werden kann, ist im Hinblick auf die Verwendung von mathematischen Begriffen und der genutzten Formelsprache nachzuvollziehen. Unter der sprachlichen Betrachtung von mathematischen Lehrund Lernprozessen nimmt Sprache als Lerngegenstand die potenziell dominanteste Rolle im Mathematikunterricht ein, da sie am deutlichsten erscheint. In der Diskussion der kognitiven Funktion in Abschnitt 2.3.1 wurde beschrieben, inwieweit Sprache die Konzeption von Begriffen durch Erfahrungen, Verknüpfungen und Darstellungen prägt. Für mathematische Begriffe ist ein besonderer Stimulus der, dass die begrifflichen Repräsentationen von mathematischen Objekten eine präzise sprachliche Darstellung erfordern, was schließlich zu einer starken Formalisierung von Sprache führt (Freudenthal, 1973). Die Möglichkeit, im Mathematikunterricht die Spezifika der mathematischen Sprache im Sinne des Lerngegenstandes kennenzulernen, ermöglicht es Lernenden, an der Sprachgemeinschaft der Mathematikerinnen und Mathematiker teilzunehmen (Meyer \& Tiedemann, 2017).

Fachsprachliche Begriffe sind im Lernprozess dabei oft bedeutende Elemente, die zum Verstehen oder zur Weiterentwicklung von mathematischen Inhalten notwendig sind. So dient die relative Häufigkeit als begriffliches Konzept für viele weitere mathematische Inhalte wie dem Verständnis der LaplaceWahrscheinlichkeit. H. Meier und Schweiger (1999) beschreiben mathematische Begriffe als Vernetzung von Wissen über mathematische Objekte, Beziehungen, Operationen und Strukturen und orientieren sich dabei an der kognitiven Funktion von Sprache.

Es lassen sich viele Formen von mathematischen Begriffen, Lehrsätzen und Verfahren unterscheiden. Dies demonstriert, welchen Stellenwert Sprache als Lerngegenstand im Mathematikunterricht besitzt. Neben inhaltsbezogenen Begriffen aus den unterschiedlichen Domänen der Mathematik sind Relationsbegriffe wie ist kleiner als, Operationsbegriffe und Strukturbegriffe (Stellenwertsystem) zentrale Merkmale von Begriffsverwendungen im Mathematikunterricht $(\mathrm{H}$. Maier \& Schweiger, 1999).

Die Mächtigkeit der Sprache als Lerngegenstand deutet sich im Bereich der Kenntnisse von Sätzen und Verfahren an. So dienen explizite, begrifflich fixierte Hinweise dazu, auf die Verwendung von Verfahren als Lösungs- bzw. Strukturierungshilfe aufmerksam zu machen - etwa, wenn bei der Vereinfachung von Termen der Hinweis der Verwendung der binomischen Formeln angegeben ist. 
Neben dem Wissen über die begrifflichen Gegenstände im Mathematikunterricht, die als „Produktziele“ bezeichnet werden können (H. Maier \& Schweiger, 1999, S. 59), sind Kenntnisse über allgemeine mathematische Prozesse Teil von Sprache als Lerngegenstand. Bauer (1978) beschreibt unterschiedliche Prozessziele für die Aktivität des mathematischen Arbeitens, beispielsweise klassifizieren, generalisieren und abstrahieren, systematisieren, lokales und globales Ordnen, definieren und beweisen. Einerseits sind die mathematischen Prozesse in das Lernen von Mathematik einzubeziehen und sind außerdem durch die Standards für die Lehrerbildung fester Bestandteil der Konzeption von Unterricht (Kultusministerkonferenz, 2003). Andererseits ist mit den Prozesszielen die spezifische Verwendung von Sprache und Sprachhandlungen im Mathematikunterricht verbunden (vgl. Abschnitt 3.2).

\subsubsection{Lernmedium}

Sprache ist ein Medium der Vermittlung. Dieser Vermittlungsaspekt wird im Mathematikunterricht in unterschiedlicher Ausprägung realisiert. Kommunikation im Klassenzimmer bedeutet nicht nur intersubjektive Kommunikation, sondern ist bei der Behandlung des Unterrichtsinhaltes stets mit dem Objekt verknüpft, was zur Mitteilung eine (besondere) Art der sprachlichen Ausdrucksform erfordert. Da sich der Mathematikunterricht an der Wissenschaftlichkeit seiner Mutterdisziplin Mathematik ausrichtet, zeigt sich im Kommunikationsraum der Mathematikklasse die Orientierung an den unterschiedlichen Ausprägungen des fachlichen Diskurses der Mathematik - am deutlichsten geprägt durch die Verwendung der symbolischen und numerischen Notation im Mathematikunterricht.

Unter der Perspektive der Wissenschaftlichkeit ergeben sich für die Kommunikation bestimmte kommunikative Parameter, die einen wissenschaftlichen Diskursrahmen bilden und diesen von Diskursen im Alltag abgrenzen (Koch \& Oesterreicher, 2007). In Abbildung 2.1 ist die Modellierung der konzeptuell gesprochenen und geschriebenen Sprache von Koch und Oesterreicher (2007) dargestellt. Konzeptuell mündlich sind jene Kommunikationsformen, die sich an den Parametern der Privatheit, der Vertrautheit der Kommunikationspartnerinnen und -partner, der emotionalen Beteiligung, der Face-to-Face-Kommunikation, der Dialogizität, der Spontanität etc. orientieren. Es handelt sich um Sprachhandlungen, mit denen Lernende in ihrer Lebenswirklichkeit vertraut sind. Konzeptuell schriftliche Kommunikationsformen zeichnen sich im Gegensatz dazu u. a. durch die Parameter der Öffentlichkeit, Fremdheit der Kommunikationsteilnehmenden, geringen emotionalen Beteiligung, raum-zeitlichen Distanz, Monologizität und Reflektiertheit aus (Koch \& Oesterreicher, 2007). 


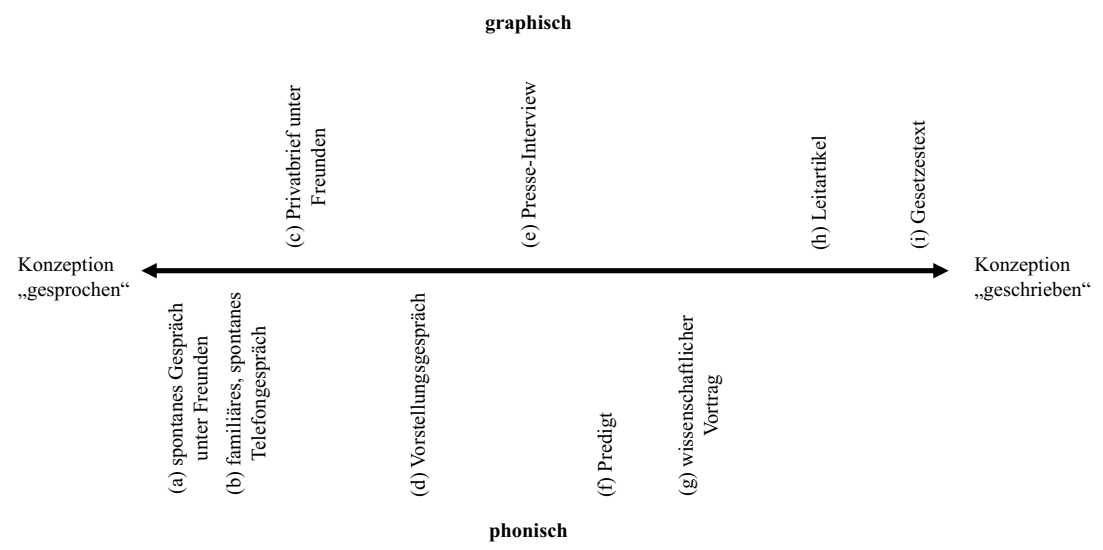

Abbildung 2.1 Kommunikationsformen auf dem konzeptionellen Kontinuum nach Koch und Oesterreicher (2007, S. 349)

Es sind Sprachhandlungen, die durch familiäre Strukturen geprägt sein können, die jedoch für den Unterricht nicht vorausgesetzt werden können. In Abbildung 2.1 ist zum einen zu erkennen, dass sich die Konzeption der Kommunikation bei konzeptuell mündlichen Formen nicht alleine auf phonische Aspekte reduzieren lässt. So ist Kommunikation per WhatsApp ein Beispiel für eine grafische Kommunikationsform, die konzeptuell schriftlich ist. Zur Kennzeichnung der „,medialen Dichotomie“ werden die Begriffe der Nähe und der Distanz verwendet (Koch \& Oesterreicher, 2007, S. 350). Im Mathematikunterricht werden aufgrund der erwähnten Nähe zur Fachwissenschaft Mathematik Aspekte der Distanz verwendet. So sind beispielsweise Klassendialoge im Kontext von Ritualen und pädagogischen Hinweisen tendenziell konzeptuell mündlich, während sich eine Schulbuchdefinition tendenziell als konzeptuell schriftlich erweist (Meyer \& Tiedemann, 2017).

Im Kontext der Annäherung an eine Kommunikation der Nähe und Distanz, die im Mathematikunterricht in der Interaktion und der Entwicklung der Verwendung eines fachlichen Diskurses ihren Ausdruck findet, verweist insbesondere die kommunikative Distanz auf eine zunehmende Verwendung einer habitualisierten Sprache infolge der Institutionalisierung des Lernens. Eine erste Unterscheidung diesbezüglich ist durch Cummins (1979, S. 198) erfolgt. Er differenziert zwischen „,basic interpersonal communicative skills (BICS)“ und „cognitive academic language proficiency (CALP)“. Dabei verweist BICS auf eine ,conversational 
fluency", womit allgemeine Fähigkeiten gemeint sind, sich in einer Sprache auszudrücken (Cummins, 2017, S. 60). Beispiele hierfür können der familiäre und private Kontext sein. Mit CALP werden die Fähigkeiten von Lernenden beschrieben, sowohl schriftlich als auch mündlich Konzepte und Ideen zu verstehen und auszudrücken, die im Kontext der Schule relevant sind (Cummins, 2017). Dementsprechend ist CALP eng mit der schulischen Biografie und der Verwendung der sprachlichen Handlungen verknüpft, die mit der Institution Schule assoziiert sind. So formuliert Cummins den Erwerb und die Definition von CALP unter der Perspektive der unterschiedlichen Stadien, in denen CALP erworben wird und relevant ist:

CALP or academic language proficiency develops through social interaction from birth but becomes differentiated from BICS after the early stages of schooling to reflect primarily the language that children acquire in school and which they need to use effectively if they are to progress successfully through the grades. The notion of CALP is specific to the social context of schooling, hence the term academic. Academic language proficiency can thus be defined as , the extent to which an individual has access to and command of the oral and written academic register of schooling'. (Cummins, 2017, S. 61 ff.).

Damit entwickelt sich CALP, ähnlich wie BICS, bereits ab der Geburt. Die Differenzen zwischen beiden Formen treten zu Beginn der Schulzeit auf: CALP bezieht sich auf die sprachlichen Fähigkeiten, die in der Schule benötigt werden, um erfolgreich zu sein und die als Ressource des familiären Umfelds vorhanden sein können oder nicht.

Ausgehend von den Überlegungen zu sprachlichen Fähigkeiten, die sich insbesondere im Schulkontext als zentral erweisen, ist in der deutschsprachigen Forschung der Begriff der Bildungssprache prominent. Morek und Heller (2012, S. 70) haben für diesen Terminus bedeutende Ausprägungslinien erläutert, die sich in Bildungssprache als Medium von Wissenstransfer (kommunikative Funktion), Werkzeug des Denkens (epistemische Funktion - die in dieser Arbeit als kognitive Funktion beschrieben wurde) sowie Eintritts- und Visitenkarte (sozialsymbolische Funktion) differenzieren. Bildungssprache kann als besonders relevantes Sprachmedium für den Mathematikunterricht betrachtet werden. Bildungssprache und weitere angelehnte Begriffe werden in Abschnitt 4.2 unter der Perspektive des Registerbegriffs weiter erläutert. 


\subsubsection{Lernvoraussetzungen und -hindernisse}

Sprache nimmt einen großen Teil der inhaltlichen Vermittlung im Mathematikunterricht ein. In der Konzeption von Mathematikunterricht wird der Umgang mit sprachlichen Ressourcen nicht immer mit betrachtet, was dazu führt, dass Sprache kein eigenes Lernziel für den Fachunterricht darstellt. Unter dieser Perspektive wird Sprache zur Lernvoraussetzung im Hinblick auf den Erwerb sprachlicher Mittel, die für den Fachunterricht erforderlich sind und dadurch für einige Lernende zum Lernhindernis.

Dass Lernvoraussetzungen und -hindernisse durch Sprache bestehen, unterstreichen die Erkenntnisse der empirischen Studien, die in Abschnitt 2.2 präsentiert wurden (u. a. PISA, TIMSS und IQB). Speziell zeigen sich hierbei die erwähnten Heterogenitätsmerkmale, der Migrationshintergrund und der sozioökonomische Hintergrund als Risikofaktoren für geringere mathematische Leistung. Insbesondere scheinen die im Mathematikunterricht zum Teil vorausgesetzten sprachlichen Mittel der Bildungs- und Unterrichts- bzw. Fachsprache (vgl. Abschnitt 4.2) für diese Schülerkohorte ein Hindernis zu sein. Das hängt mit den sprachlichen Kompetenzen zusammen, die bei den genannten Kohorten geringer ausfallen (Prediger et al., 2015). Eine unzureichende Lesekompetenz im Deutschen wirkt sich negativ auf die Leistungschancen in Mathematik (und naturwissenschaftlichen Fächern) aus (Gogolin \& Lange, 2011).

Damit zeigt sich eine starke Evidenz dafür, dass eine unzureichende Beherrschung der sprachlichen Fähigkeiten in Deutsch zu Leistungsdisparitäten u. a. im Mathematikunterricht führt. Ziel für den Mathematikunterricht muss es dementsprechend sein, die sprachlichen Kompetenzen zu fördern, die im Zusammenhang mit mathematischen Lernprozessen stehen, sodass sich die Diskrepanzen reduzieren. Dieser Aspekt wird in der vorliegenden Arbeit mehrfach, auch durch den Verweis auf weitere empirische Erhebungen, aufgegriffen, da er eine besondere Bedeutung für die Erstellung des Instruments für sprachliche Variationen von Textaufgaben im Mathematikunterricht hat.

\subsection{Zusammenfassung}

Sprache hat eine hohe Bedeutung für das Lernen von Mathematik. Besondere Aufmerksamkeit erhielt die Sprache durch die verstärkte Betrachtung von Heterogenitätsmerkmalen. Heterogenität ist ein fester Bestandteil der schulischen Realität und wird als Qualitätsmerkmal von Unterricht gewertet. Sprachliche Fähigkeiten beim Lernen im Mathematikunterricht können ebenfalls heterogen 
ausgeprägt sein. Studien zeigen, dass die Unterschiedlichkeit der sprachlichen Fähigkeiten zu Leistungsdisparitäten im Mathematikunterricht führen. Dahingehend ist es zwingend erforderlich, auf die Unterschiedlichkeit der sprachlichen Fähigkeiten der Lernenden im Mathematikunterricht didaktisch zu reagieren.

Sprache und die Mathematik - insbesondere das Lernen mathematischer Inhalte - haben viele unterschiedlich offensichtliche Überschneidungspunkte. Das konstruktive Prinzip mathematischen Lernens gilt ebenfalls für die damit assoziierten sprachlichen Ressourcen, die für das Lernen benötigt werden und nur mit dem Gegenstand zusammen gelernt und verwendet werden können.

In einen sprachintegrierten Mathematikunterricht müssen die unterschiedlichen Erscheinungsformen von Sprache im Fachunterricht einbezogen werden. Dies umfasst die funktionalen Grundlagen von Sprache, die bei der Konzeption eines sprachintegrierten Mathematikunterrichts mitgedacht werden sollten. Sowohl die kognitive und kommunikative Funktion als auch die metafunktionale Perspektive sollten fachsprachlich einbezogen werden, damit die Struktur der Kommunikation im Mathematikunterricht verständlich wird. Ebenfalls relevant ist die Kenntnis der drei unterschiedlichen Aspekte von Sprache im Mathematikunterricht: Sprache als Lerngegenstand zum Verständnis der sprachlichen Grundlagen, als Lernmedium zum Verständnis des Vermittlungsgedankens und als Lernvoraussetzung und Lernhindernis zum Verständnis der Notwendigkeiten und Schwierigkeiten, die Sprache im Mathematikunterricht mit sich bringen kann. Mithin dient ein stärkerer Fokus auf die Potenziale der sprachlichen Vermittlung dazu, eine adäquate Lehre zu garantieren, da die Aspekte deutlich machen, dass eine fachliche Vermittlung nicht ohne Sprache funktioniert. Dahingehend sollten, unter praktischen Gesichtspunkten, neben den fachlichen Lernzielen auch lexikalische Lernziele für die Unterrichtsentwicklung mitbetrachtet werden.

Ausblick: Die theoretischen und empirischen Erkenntnisse erklären die Bedeutung von Sprache im Allgemeinen. Insofern ist eine weitere Spezifizierung notwendig. Aufgrund des Ziels der Arbeit wurde der abgeleitete Fokus gesetzt, Texte im Mathematikunterricht zu betrachten. Aus diesem Grund werden im folgenden Kapitel 3 Texte und die mit den Texten in Beziehung stehenden Kontexte im Mathematikunterricht betrachtet. 
Open Access Dieses Kapitel wird unter der Creative Commons Namensnennung 4.0 International Lizenz (http://creativecommons.org/licenses/by/4.0/deed.de) veröffentlicht, welche die Nutzung, Vervielfältigung, Bearbeitung, Verbreitung und Wiedergabe in jeglichem Medium und Format erlaubt, sofern Sie den/die ursprünglichen Autor(en) und die Quelle ordnungsgemäß nennen, einen Link zur Creative Commons Lizenz beifügen und angeben, ob Änderungen vorgenommen wurden.

Die in diesem Kapitel enthaltenen Bilder und sonstiges Drittmaterial unterliegen ebenfalls der genannten Creative Commons Lizenz, sofern sich aus der Abbildungslegende nichts anderes ergibt. Sofern das betreffende Material nicht unter der genannten Creative Commons Lizenz steht und die betreffende Handlung nicht nach gesetzlichen Vorschriften erlaubt ist, ist für die oben aufgeführten Weiterverwendungen des Materials die Einwilligung des jeweiligen Rechteinhabers einzuholen.

(c) (9) 